\title{
Hyperinflation is Associated with Increased Respiratory Rate and is a More Sensitive Measure of Cystic Fibrosis Lung Disease During Infancy Compared to Forced Expiratory Measures
}

\author{
Heather Muston ${ }^{1}$, James Slaven ${ }^{2}$, Christina Tiller ${ }^{3}$, Charles Clem ${ }^{3}$, Thomas Ferkol ${ }^{4}$, Sarath \\ Ranganathan ${ }^{5}$, Stephanie Davis ${ }^{6}$, and Clement Ren ${ }^{7}$ \\ ${ }^{1}$ Riley Hospital for Children at Indiana University Health \\ ${ }^{2}$ Indiana University School of Medicine \\ ${ }^{3}$ James Whitcomb Riley Hospital for Children \\ ${ }^{4}$ Washington University in Saint Louis School of Medicine \\ ${ }^{5}$ Royal Children's Hospital, Melbourne \\ ${ }^{6}$ University of North Carolina at Chapel Hill School of Medicine \\ ${ }^{7}$ Indiana University System
}

October 26, 2020

\begin{abstract}
ABSTRACT Background: The goal of this study was to identify clinical features associated with abnormal infant pulmonary function tests (iPFTs), specifically functional residual capacity (FRC), in infants with cystic fibrosis (CF) diagnosed via newborn screen (NBS). We hypothesized that poor nutritional status in the first 6-12 months would be associated with increased FRC at 12-24 months. Methods: This study utilized a combination of retrospectively and prospectively collected data from ongoing research studies and iPFTs performed for clinical indications. Demographic and clinical features were obtained from the electronic medical record. Forced expiratory flows and volumes were obtained using the raised volume rapid thoracoabdominal technique (RVRTC) and FRC was measured via plethysmography. Results: A total of 45 CF NBS infants had iPFTs performed between 12-24 months. Mean forced vital capacity, forced expiratory volume in 0.5 second, and forced expiratory flows were all within normal limits. In contrast, the mean FRC z-score was $2.18(95 \% \mathrm{CI}=1.48,2.88)$ and the mean respiratory rate $(\mathrm{RR})$ z-score was $1.42(95 \% \mathrm{CI}=0.95,1.89)$. There was no significant association between poor nutritional status and abnormal lung function. However, there was a significant association between higher RR and increased FRC, and a RR cutoff of 36 breaths/min resulted in $92 \%$ sensitivity to detect hyperinflation with $32 \%$ specificity. Conclusions: These results suggest that FRC is a more sensitive measure of early CF lung disease than RVRTC measurements and that RR may be a simple, non-invasive clinical marker to identify CF NBS infants with hyperinflation.
\end{abstract}

\section{INTRODUCTION}

Cystic fibrosis $(\mathrm{CF})$ is the most common life-limiting genetic disease in Caucasians and pulmonary disease remains the primary etiology of morbidity and mortality in CF. Lung disease in CF is present during infancy and events early in life play a critical role in long-term outcomes. Early Pseudomonas aeruginosa (Pa) infection, elevated free neutrophil elastase in bronchoalveolar lavage fluid, history of wheeze and history of hospitalizations are some risk factors in infancy and early childhood associated with lower lung function and/or bronchiectasis in later childhood ${ }^{1-4}$. Nutritional status within the first years of life predicts lung function in later childhood ${ }^{4-6}$. Despite the advent of newborn screening (NBS), CF lung disease continues to occur in the youngest population; however, it is often present without symptoms ${ }^{7-11}$. Given that lung 
function in infancy tracks into preschool years ${ }^{12-14}$, identifying sensitive clinical markers of early disease is imperative. Early identification will lead to treatment and intervention, ultimately preventing or mitigating CF lung disease.

The ability to assess lung disease during infancy remains challenging ${ }^{15-17}$. Chest computed tomography is a sensitive measure of early CF lung disease, but requires sedation and exposure to radiation ${ }^{1,18}$. Infant pulmonary function tests (iPFTs) detect early lung disease but require sedation and a high level of technical skill ${ }^{16,19}$. The success rate of obtaining forced expiratory flows (FEF) via the raised volume rapid thoracoabdominal technique (RVRTC) in multicenter clinical trials is not as high as obtaining functioning residual capacity via plethysmography $\left(\mathrm{FRC}_{\text {pleth }}\right)^{16,17,19}$. Although $\mathrm{FRC}_{\text {pleth }}$ has been measured in studies of infants with $\mathrm{CF}$, data from infants diagnosed via NBS are more limited, with only one other cohort previously described ${ }^{7,16,20,21}$. Given that early CF lung disease begins in the small peripheral airways, FRC may be a more sensitive measure of early CF lung disease when compared to forced expiratory flows. In CF NBS infants, there is limited information correlating FRC with clinically useful indicators of early lung disease.

The objective of our study was to identify clinical features associated with abnormal lung function, specifically FRC, in CF NBS infants. We hypothesized that poor nutritional status in the first 6-12 months of life is associated with more hyperinflation at 12-24 months of age.

\section{METHODS}

\section{Study Design}

This study utilized a combination of retrospectively and prospectively collected data. The retrospective data were acquired from 23 infants who had iPFTs performed at Riley Hospital for Children from 2012-2018 as part of clinical care and 12 infants who underwent iPFTs as part of other research studies (ClinicalTrials.gov NCT01973192 and NCT04026360). We prospectively recruited an additional 10 infants followed at the CF Center at Riley Hospital for lung function testing, bringing the total sample size to 45 infants.

The inclusion criteria were (1) an age at time of iPFTs of 12-24 months of age and (2) a diagnosis of CF through NBS ${ }^{22}$. Exclusion criteria included gestational age less than 36 weeks. The study was approved by the Indiana University Institutional Review Board and informed written consent was obtained from the parents of prospectively recruited infants.

Clinical data, including mutation analysis, ethnicity, respiratory cultures, antibiotic and systemic steroid courses, smoke exposure, history of wheezing and nutritional indices, were obtained from the electronic medical record. Nutritional status indices obtained for this study were weight-for-age (WFA) and weightfor-length (WFL) z-scores based on the Centers for Disease Control growth charts during the first 24 months of life ${ }^{23}$. Weight and crown-heel lengths were obtained during CF clinic visits and during iPFT testing.

\section{Measurement of Lung Function}

All iPFTs were performed using the Jaeger BabyBody device (CareFusion, San Diego, California) equipment. Infants were sedated with $50-100 \mathrm{mg} / \mathrm{kg}$ oral chloral hydrate and cardiopulmonary monitoring was performed throughout testing. FRC was measured using plethysmography ${ }^{24}$. Forced expiratory flows were obtained using the RVRTC technique. All lung function parameters were obtained according to published guidelines 24,25 . Infant PFT values were converted to z-scores based on previously published reference equations ${ }^{26,27}$. Respiratory rate data were obtained from tidal breathing analysis performed during iPFT testing for a minimum period of $20-30$ breaths ${ }^{27}$ or from the vital signs flowchart. Only iPFT data that met published quality control criteria were used for analysis ${ }^{24,25}$.

\section{Statistics}

Data were examined for calculation and distribution for statistical analysis SAS v9.4 (SAS institute, Cary, NC). A power analysis performed prior to the study showed that with a sample size of 44 , an alpha level of 0.05 and power [?] 0.80 , we could detect a significant association between the clinical variables with iPFT z-scores when the correlation was as low as 0.41. Statistical significance was defined as p [?] 0.05. For 
categorical variables, frequency and percentage analysis were performed. For continuous iPFT variables, mean and 95\% confidence intervals were calculated. Chi-square analysis was used to evaluate the proportion of infants in each group with abnormal FRC vs. forced expiratory volume in 0.5 seconds $\left(\mathrm{FEV}_{0.5}\right)$ parameters. To determine the association between WFA/WFL z-score and lung function, Pearson correlation coefficients were calculated. Associations between lung function and categorical clinical risk factors were calculated using Student's t-test or Mann-Whitney U test as appropriate. Correlation between FRC and respiratory rate $(\mathrm{RR})$ was determined using Spearman rank-sum correlation coefficients. We conducted additional analyses stratifying infants into normal versus obstruction or hyperinflation. For these analyses, we defined obstruction as an $\mathrm{FEV}_{0.5}$ z-score $<-2$ and hyperinflation as $\mathrm{FRC}$ z-score $>2$. To identify the respiratory rate cutoff that yielded the best combination of sensitivity and specificity in predicting hyperinflation, we used logistic regression with Youden's Index.

\section{RESULTS}

\section{Study Population}

Figure 1 shows the derivation of the study cohort. A total of 45 infants were included in this study. Of the 57 patients with previous iPFTs, 35 met our inclusion and exclusion criteria. Fourteen infants followed at the CF Center at Riley were eligible for iPFTs for the prospective cohort during the study period and the parents of 12 infants (87\%) agreed to testing. Eleven infants ultimately underwent iPFT, of which $10(91 \%)$ yielded research-quality data. Clinical characteristics of the cohort are described in Table 1. The majority of infants were Caucasian (95.6\%) and had homozygous Class I, II or III mutations (93.1\%). The most common respiratory infection detected was methicillin-sensitiveStaphylococcus aureus (66.7\%) and $35.6 \%$ of the cohort had a history of at least one positive Pa respiratory culture. Oral antibiotic use in the first year was high, with $91.1 \%$ of infants receiving at least one course and a median of 2 courses of antibiotics.

\section{Lung Function Data}

Research-quality FRC and $\mathrm{FEV}_{0.5}$ data were obtained in $96 \%$ and $100 \%$ of infants, respectively. As demonstrated in Table 2, the forced vital capacity (FVC) z-score was decreased, but all other RVRTC data were within normal limits. In contrast to the RVRTC data, the mean FRC z-score was significantly elevated at 2.18. Figure 2 demonstrates that the proportion of patients with hyperinflation (44\%) was significantly higher than infants with diminished $\mathrm{FEV}_{0.5}(7 \%)(\mathrm{p}<0.001)$. Mean RR was also significantly elevated, and correlated with FRC (Figure 3) with a Spearmen correlation coefficient of $0.377(\mathrm{p}=0.01)$. Using logistic regression to calculate Youden's index, we found that a RR cutoff of 36 breaths/min resulted in $92 \%$ sensitivity to detect hyperinflation with $32 \%$ specificity.

\section{Risk Factors for Abnormal Lung Function}

We next examined the association between clinical risk factors and abnormal lung function (Table 3). No significant association was seen between WFA or WFL z-scores at 3 months of age, 6 months of age or 6 months prior to iPFT and lung function z-scores. No significant association was seen between WFA or WFL z-score at 6 months of age and raw lung function values (data not shown). We did not observe any significant association between clinical risk factors and hyperinflation. Consistent with previously reported literature $^{3,28}$, a history of wheezing $(\mathrm{p}=0.02)$ and smoke exposure $(\mathrm{p}=0.05)$ were associated with diminished $\mathrm{FEV}_{0.5}$.

\section{DISCUSSION}

In this mixed cohort of retrospectively and prospectively collected iPFT data from CF NBS infants, we found that hyperinflation was more common than diminished $\mathrm{FEV}_{0.5}$ and FEF. Furthermore, RR was elevated and correlated with increased FRC. These results suggest that FRC is a more sensitive measure of early CF lung disease and that measurement of RR may be a simple, non-invasive clinical marker to identify CF NBS infants with hyperinflation.

Although there have been several iPFT studies in CF NBS infants, few have reported FRC data. Some of the 
FRC measurements in previous studies were performed using multiple breath washout (MBW), which may underestimate hyperinflation compared to plethysmography ${ }^{29}$. In a multicenter iPFT study of CF infants primarily diagnosed clinically, hyperinflation was more common than diminished $\mathrm{FEV}_{0.5}{ }^{16}$. Our results are similar, suggesting that early diagnosis of CF through NBS does not prevent hyperinflation. The only other study to report $\mathrm{FRC}_{\text {pleth }}$ in CF NBS infants has been from the London Cystic Fibrosis Collaboration ${ }^{7,9,11}$. FRC was elevated in this Collaborative, but hyperinflation was less common and obstruction, as measured by $\mathrm{FEV}_{0.5}$, was more common in this group when compared to our cohort. Our finding that hyperinflation was much more common than decreased $\mathrm{FEV}_{0.5}$ in CF NBS infants highlights that small airway obstruction is present in early life. It is also consistent with chest computed tomography findings that demonstrate that air trapping is the earliest and most prevalent sign of lung disease in CF NBS infants ${ }^{2}$.

Very few studies have reported RVRTC and FRC data in CF NBS infants in the USA. In our study, there was no significant difference in $\mathrm{FEV}_{0.5}$ or $\mathrm{FEF}$ compared to normal reference values ${ }^{7,8,30}$. Britton, et al reported normal $\mathrm{FEV}_{0.5}$ and FEF in CF NBS infants born in the USA ${ }^{31}$. Further, Pollak, et al reported that a large proportion of infants had normal $\mathrm{FEV}_{0.5}$ and $\mathrm{FVC}$ in infancy and these parameters did not predict abnormal lung function in early school-age spirometry ${ }^{32}$. This further suggests that forced expiratory flows and volumes are less sensitive measures of early CF lung disease.

Elevated RR in children and infants with CF has been described previously ${ }^{33-35}$. These studies have not shown an association between RR and FEF or tidal breathing indices, although one study did report an association between elevated RR and increased lung clearance index (LCI) in infants with $\mathrm{CF}^{35}$. However, this study did report an association between RR and FRC obtained via MBW, which may underestimate FRC $_{\text {pleth }}$. They also did not define RR thresholds associated with abnormal LCI. With a cutoff RR of 36, we demonstrated a high sensitivity for identifying infants with hyperinflation as measured through $\mathrm{FRC}_{\mathrm{pleth}}$, a sign of early lung disease. In contrast to measurement of $\mathrm{FRC}_{\text {pleth }}$ in infants, which requires a high degree of technical skill and expensive equipment, measurement of RR is much simpler, non-invasive and does not require sedation, suggesting it may be a clinically useful marker of infants at an increased risk of more severe early lung disease.

Similar to previous studies, we found that wheezing and second-hand smoke exposure were associated with worse lung function ${ }^{3,28}$. A history of $\mathrm{Pa}$ infection was also associated with lower lung function, but this was not statistically significant in our cohort. Several studies have shown a relationship between early nutritional status and lung function at 6-12 years ${ }^{5,6,36}$, and similar to other iPFT studies we observed lower WFA z-scores in our cohort 7,8 . However, we were unable to demonstrate that lower nutritional indices in early infancy were associated with lower lung function later in infancy. Potential reasons for this include the possibility that $\mathrm{FEF}$ and $\mathrm{FRC}_{\text {pleth }}$ are not sensitive enough to detect a significant association or that 6 months was an insufficient time between lung function and the measured outcomes to detect a significant association.

Our results have potential implications for clinical care and research. Hyperinflation was significantly more common than decreased FEF and $\mathrm{FEV}_{0.5}$, suggesting that it may be a more sensitive measure of early lung disease and could serve as an additional sensitive measure for research studies evaluating early infant CF lung disease. $\mathrm{FRC}_{\text {pleth }}$ requires less technical skill compared to FEF. RR correlated significantly with hyperinflation. Since RR can easily be obtained without the need for specialized equipment, our results suggest that RR could serve as a clinical marker for early $\mathrm{CF}$ lung disease. However, the role of RR monitoring in $\mathrm{CF}$ infants needs further study before it can be used in routine clinical practice.

There are several limitations to our study. This is a single-center study, which could limit generalizability. However, our cohort had similar clinical characteristics compared to other CF NBS studies. Our sample size was also comparable to other iPFT studies in CF infants ${ }^{7-9,32}$. Some of our iPFT data came from clinically indicated tests, which may have biased our study cohort towards greater disease severity. To help reduce selection bias, we recruited a cohort of clinically mild infants and asymptomatic infants who had iPFTs as part of other research studies. Although the cohort may still have been biased towards more severe disease, this would not have affected the association between RR and FRC. RR was measured during sedation with 
chloral hydrate. While this may have altered the actual respiratory rate numbers, it should not have affected the correlation we demonstrated. As with all iPFT studies, there is a paucity of normal reference data and we relied on the same data utilized by other studies. Although our reference values for FRC were obtained from a different study from which we obtained our $\mathrm{FVC}, \mathrm{FEV}_{0.5}, \mathrm{FEF}_{25-75}$, and $\mathrm{FEF}_{75}$ reference values, they were all performed on the same equipment using the same methods and sedation protocol.

In summary, this study of lung function in CF NBS infants found that hyperinflation is more common than decreased FEF, and that RR correlated with FRC. These results have potential implications for the care and study of early CF lung disease in infants. Further research is needed to evaluate the role of RR measurement as a non-invasive, simple and clinically useful marker of early CF lung disease.

\section{ACKNOWLEDGEMENTS}

The authors thank Drs DB Sanders and M Bozic for their advice and guidance during the study. Research funded through a CF Foundation Clinical Fellowship grant (MUSTON18A0-D3-SL), Indiana University CTSI grant (UL1TR001108), CF Foundation grant DAVIS08Y2 and NIH grant 5R01HL116211-03 to S Davis, T Ferkol and S Ranganathan.

Figure Legends:

\section{Figure 2}

${ }^{*}$ Circled area indicates those infants with hyperinflation but no significant obstruction

\section{Figure 3}

*Spearman Correlation Coefficient 0.377; $\mathrm{p}=0.01$

Table 1: Characteristics of the Cohort.

\begin{tabular}{ll}
\hline Characteristic & \\
\hline Mutation homozygous class I or II (N) & $41(93.1)$ \\
Pancreatic insufficient (N) & $43(95.6)$ \\
Male (N) & $20(44.4)$ \\
Caucasian (N) & $43(95.6)$ \\
Hospital Admission prior to iPFT (N) & $20(44.4)$ \\
Age at iPFT testing in months (Mean, SD) & $15.8(3.16)$ \\
WFA z-score at time of iPFT (Mean, SD) & $-0.67(0.93)$ \\
Height z-score at time of iPFT (Mean, SD) & $-0.4(0.98)$ \\
WFL z-score at time of iPFT (Mean, SD) & $-0.01(0.83)$ \\
Second hand smoke exposure (N) & $15(33.3)$ \\
Oral corticosteroid treatment in first year of life (N) & $11(24.4)$ \\
Oral antibiotics in first year of life (N) & $41(91.1)$ \\
Number oral antibiotics before iPFT (Median, range) & $2(0-10)$ \\
MSSA positive culture* (N) & $30(66.7)$ \\
MRSA positive culture* (N) & $6(13.3)$ \\
Pseudomonas aeruginosa positive culture* (N) & $16(35.6)$ \\
Stenotrophomonas maltophilia positive culture* (N) & $8(17.8)$ \\
WFA z-score at 6 months of age (Mean, SD) & $-0.72(1.1)$ \\
WFL z-score at 6 months of age (Mean, SD) & $-0.51(1.03)$ \\
\hline
\end{tabular}

Data reported as Number (Percentages) unless otherwise noted.

* Reported as ever/never positive before iPFT

Table 2: Lung Function Data 


\begin{tabular}{lll}
\hline iPFT Parameter & z-score Mean $(95 \%$ CI $)$ & z-score Range \\
\hline FVC & $\mathbf{- 0 . 3 9}(-\mathbf{0 . 6 8}$ to $\mathbf{- 0 . 1})$ & $\mathbf{- 4 . 8 9}$ to $\mathbf{1 . 8 6}$ \\
FEV $_{0.5}$ & $-0.05(-0.46$ to 0.35$)$ & -5.49 to 1.82 \\
FEF $_{25 / 75}$ & $0.37(-0.05$ to 0.8$)$ & -3.25 to 3.25 \\
FEF $_{75}$ & $0.09(-0.3$ to 0.49$)$ & -3.12 to 2.68 \\
Respiratory Rate & $\mathbf{1 . 4 2}(\mathbf{0 . 9 5}$ to $\mathbf{1 . 8 9})$ & $\mathbf{- 0 . 6}$ to $\mathbf{5 . 3 3}$ \\
TV & $-0.2(-0.54$ to 0.14$)$ & -1.99 to 2.4 \\
FRC & $\mathbf{2 . 1 8}(\mathbf{1 . 4 8}$ to $\mathbf{2 . 8 8})$ & $\mathbf{- 1 . 2 1}$ to $\mathbf{9 . 4}$ \\
\hline
\end{tabular}

Table 3: Association between Clinical Risk Factors and Lung Function

\begin{tabular}{lll}
\hline & FEV $_{\mathbf{0 . 5}}$ (z-score) & FRC (z-score) \\
\hline WFA z-score at 6 months & $0.241 ; \mathrm{p}=0.12$ & $-0.012 ; \mathrm{p}=0.94$ \\
WFA z-score 6 months prior iPFT & $0.081 ; \mathrm{p}=0.61$ & $0.106 ; \mathrm{p}=0.51$ \\
WFL z-score at $\mathbf{6}$ months of age & $-0.080 ; \mathrm{p}=0.60$ & $-0.006 ; \mathrm{p}=0.97$ \\
WFL z-score 6 months prior iPFT & $-0.215 ; \mathrm{p}=0.16$ & $0.025 ; \mathrm{p}=0.87$ \\
Pseudomonas aeruginosa & & \\
No (n=29) & $0.28(1.06) ; \mathrm{p}=0.06$ & $1.82(1.59) ; \mathrm{p}=0.26$ \\
Yes (n=16) & $-0.66(1.70)$ & $2.86(3.28)$ \\
Wheezing & $0.41(0.94) ; \mathrm{p}=0.02^{*}$ & $2.05(2.40) ; \mathrm{p}=0.74$ \\
No (n=23) & $-0.60(1.62)$ & $2.30(2.37)$ \\
Yes (n=21) & & \\
Smoke Exposure & $0.21(1.05) ; \mathrm{p}=0.05^{*}$ & $2.39(2.44) ; \mathrm{p}=0.67$ \\
No (n=26) & $-0.78(1.68)$ & $2.05(2.42)$ \\
Yes (n=15) & & \\
\hline
\end{tabular}

* Pearson Correlation coefficients for continuous variables. For categorical variables, values are means (standard deviations) with p-values from Student's t-tests.

\section{References}

1. Sly PD, Gangell CL, Chen L, et al. Risk Factors for Bronchiectasis in Children with Cystic Fibrosis. New England Journal of Medicine 2013;368:1963-70.

2. Ramsey KA, Ranganathan S, Park J, et al. Early Respiratory Infection Is Associated with Reduced Spirometry in Children with Cystic Fibrosis. American Journal of Respiratory and Critical Care Medicine 2014;190:1111-6.

3. Ren CL, Konstan MW, Rosenfeld M, et al. Early childhood wheezing is associated with lower lung function in cystic fibrosis. Pediatric Pulmonology 2014;49:745-50.

4. Sanders DB, Li Z, Laxova A, et al. Risk Factors for the Progression of Cystic Fibrosis Lung Disease throughout Childhood. Annals of the American Thoracic Society 2014;11:63-72.

5. Sanders DB, Fink A, Mayer-Hamblett N, et al. Early Life Growth Trajectories in Cystic Fibrosis are Associated with Pulmonary Function at Age 6 Years. J Pediatr 2015;167:1081-8 e1.

6. Konstan M, Butler SM, Wohl MEB, et al. Growth and nutritional indexes in early life predict pulmonary function in cystic fibrosis. The Journal of Pediatrics 2003;142:624-30.

7. Nguyen TT-D, Thia LP, Hoo A-F, et al. Evolution of lung function during the first year of life in newborn screened cystic fibrosis infants. Thorax 2014;69:910-7. 
8. Linnane BM, Hall GL, Nolan G, et al. Lung function in infants with cystic fibrosis diagnosed by newborn screening. Am J Respir Crit Care Med 2008;178:1238-44.

9. Hoo A-F, Thia LP, Nguyen TTD, et al. Lung function is abnormal in 3-month-old infants with cystic fibrosis diagnosed by newborn screening. Thorax 2012;67:874-81.

10. Sly PD, Brennan S, Gangell C, et al. Lung Disease at Diagnosis in Infants with Cystic Fibrosis Detected by Newborn Screening. American Journal of Respiratory and Critical Care Medicine 2009;180:146-52.

11. Davies G, Stocks J, Thia LP, et al. Pulmonary function deficits in newborn screened infants with cystic fibrosis managed with standard UK care are mild and transient. European Respiratory Journal 2017;50.

12. Marostica PJC, Weist AD, Eigen H, et al. Spirometry in 3- to 6-Year-Old Children with Cystic Fibrosis. American Journal of Respiratory and Critical Care Medicine 2002;166:67-71.

13. Ranganathan SC, Stocks J, Dezateux C, et al. The Evolution of Airway Function in Early Childhood Following Clinical Diagnosis of Cystic Fibrosis. American Journal of Respiratory and Critical Care Medicine 2004;169:928-33.

14. N. HA, E. RW, M. ZJ, E. MC. Longitudinal assessment of lung function from infancy to childhood in patients with cystic fibrosis. Pediatric Pulmonology 2009;44:330-9.

15. Rosenfeld M, Allen J, Arets BH, et al. An official American Thoracic Society workshop report: optimal lung function tests for monitoring cystic fibrosis, bronchopulmonary dysplasia, and recurrent wheezing in children less than 6 years of age. Ann Am Thorac Soc 2013;10:S1-S11.

16. Davis SD, Rosenfeld M, Kerby GS, et al. Multicenter evaluation of infant lung function tests as cystic fibrosis clinical trial endpoints. Am J Respir Crit Care Med 2010;182:1387-97.

17. Davis SD, Ratjen F, Brumback LC, et al. Infant lung function tests as endpoints in the ISIS multicenter clinical trial in cystic fibrosis. Journal of Cystic Fibrosis 2016;15:386-91.

18. Davis SD, Fordham LA, Brody AS, et al. Computed Tomography Reflects Lower Airway Inflammation and Tracks Changes in Early Cystic Fibrosis. American Journal of Respiratory and Critical Care Medicine 2007;175:943-50.

19. Matecki S, Kent L, de Boeck K, et al. Is the raised volume rapid thoracic compression technique ready for use in clinical trials in infants with cystic fibrosis? Journal of Cystic Fibrosis 2016;15:10-20.

20. Rosenfeld M, Farrell PM, Kloster M, et al. Association of lung function, chest radiographs and clinical features in infants with cystic fibrosis. European Respiratory Journal 2013;42:1545-52.

21. Beardsmore CS. Lung function from infancy to school age in cystic fibrosis. Arch Dis Child 1995;73:51923.

22. Farrell PM, White TB, Ren CL, et al. Diagnosis of Cystic Fibrosis: Consensus Guidelines from the Cystic Fibrosis Foundation. The Journal of Pediatrics 2017;181:S4-S15.e1.

23. Kuczmarski RJ OC, Guo SS, et al. 2000 CDC growth charts for the United States: Methods and development. National Center for Health Statistics Vital Health Stat 2002;11.

24. Stocks J, Godfrey S, Beardsmore C, Bar-Yishay E, Castile R. Plethysmographic measurements of lung volume and airway resistance. European Respiratory Journal 2001;17:302-12.

25. ATS/ERS Statement: Raised Volume Forced Expirations in Infants. American Journal of Respiratory and Critical Care Medicine 2005;172:1463-71.

26. Lum S, Bountziouka V, Wade A, et al. New reference ranges for interpreting forced expiratory manoeuvres in infants and implications for clinical interpretation: a multicentre collaboration. Thorax 2016;71:27683. 
27. Nguyen TTD, Hoo A-F, Lum S, Wade A, Thia LP, Stocks J. New reference equations to improve interpretation of infant lung function. Pediatric Pulmonology 2013;48:370-80.

28. Ong T, Schechter M, Yang J, et al. Socioeconomic Status, Smoke Exposure, and Health Outcomes in Young Children With Cystic Fibrosis. Pediatrics 2017;139:e20162730.

29. Dahlqvist M, Hedenstierna G. Lung volumes measured by helium dilution and by body plethysmography with mouth and oesophageal pressures: a comparative study in patients with lung disease. Clin Physiol $1985 ; 5: 179-87$.

30. Pillarisetti N, Williamson E, Linnane B, et al. Infection, Inflammation, and Lung Function Decline in Infants with Cystic Fibrosis. American Journal of Respiratory and Critical Care Medicine 2011;184:75-81.

31. Britton LJ, Oates GR, Oster RA, et al. Risk stratification model to detect early pulmonary disease in infants with cystic fibrosis diagnosed by newborn screening. Pediatr Pulmonol 2016;51:1168-76.

32. Pollak M, Shaw M, Balkovec S, et al. Infant spirometry as a predictor of lung function at early childhood in cystic fibrosis patients. Journal of Cystic Fibrosis 2020:2116.

33. Ranganathan SC, Goetz I, Hoo A-F, Lum S, Castle R, Stocks J. Assessment of tidal breathing parameters in infants with cystic fibrosis. European Respiratory Journal 2003;22:761-6.

34. Lum S, Gustafsson P, Ljungberg H, et al. Early detection of cystic fibrosis lung disease: multiple-breath washout versus raised volume tests. Thorax 2007;62:341-7.

35. Korten I, Kieninger E, Yammine S, et al. Respiratory rate in infants with cystic fibrosis throughout the first year of life and association with lung clearance index measured shortly after birth. Journal of Cystic Fibrosis 2019;18:118-26.

36. Lai HJ, Shoff SM, Farrell PM. Recovery of Birth Weight z Score Within 2 Years of Diagnosis Is Positively Associated With Pulmonary Status at 6 Years of Age in Children With Cystic Fibrosis. Pediatrics 2009;123:714-22. 


\section{Figure 1: Derivation of the Study Cohort}

\section{Prospective Cohort}

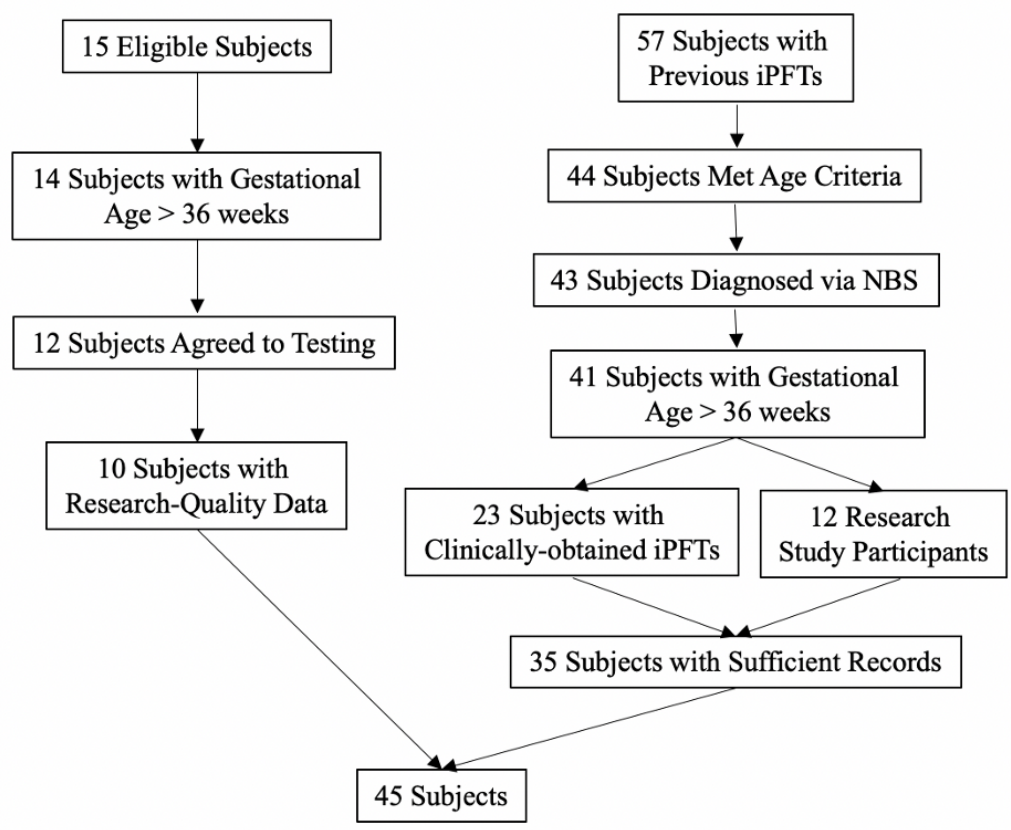

Figure 2: FEV 0.5 z-score versus FRC z-score

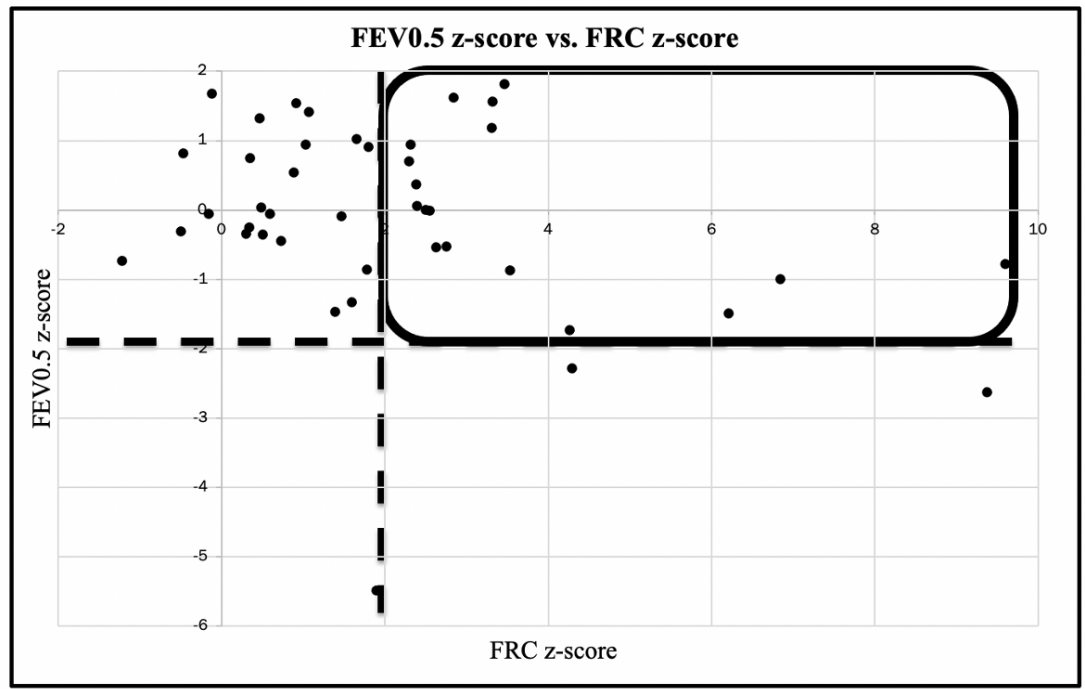


Figure 3: Association between Hyperinflation and Respiratory Rate

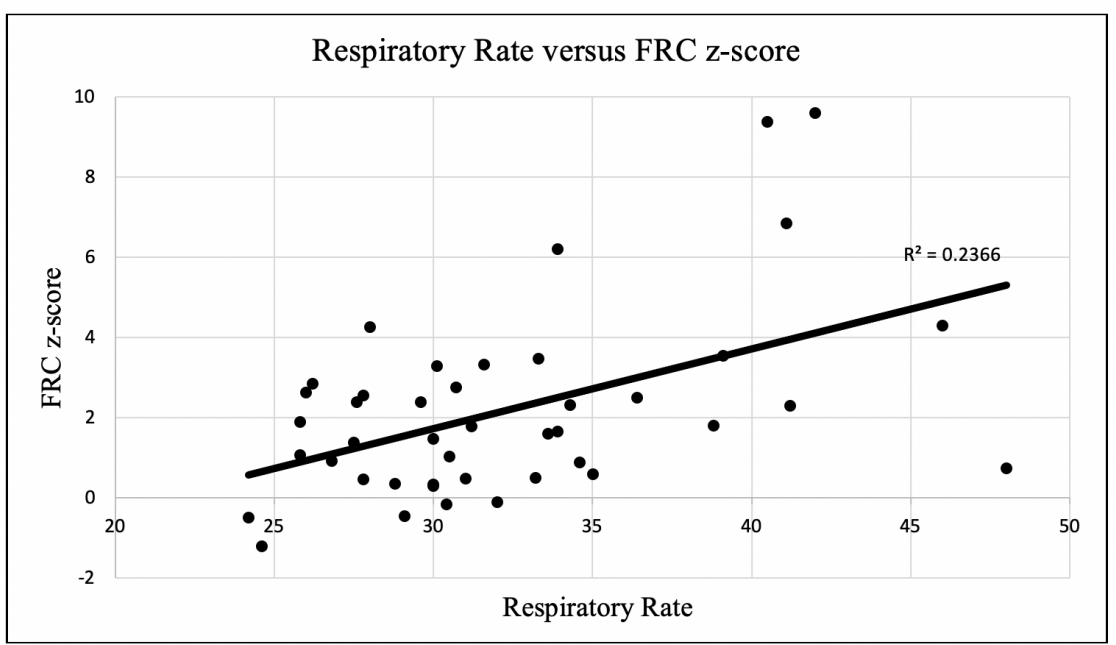

